\title{
The role of pulmonary CT scans for children during the COVID-19 pandemic
}

\author{
Ian P. Sinha $a^{1,2^{*}}$ and Musa Kaleem ${ }^{1}$
}

Keywords: COVID-19, Imaging, Children, Diagnosis

\section{Background}

It is now well established that children are significantly less likely to develop COVID-19 than adults and that they rarely suffer from severe infection [1]. One area of uncertainty, however, is how best to diagnose children with COVID-19. The accuracy of reverse transcriptase polymerase chain reaction (RT-PCR) is variable and depends on the type of sample taken, the technique used, and when during an infection it is tested $[2,3]$. There is an increasing body of literature supporting the use of high-resolution computed tomography (HRCT) scanning for adults suspected of having COVID-19 in selected situations [4], particularly those with severe illness [5].

\section{What is the role of $\mathrm{CT}$ imaging in children with COVID-19?}

The linked paper brings useful insight into the role of CT for children with COVID-19 and raises important questions [6]. This retrospective, single-centre study from Wuhan describes the use of routine chest CT scanning in 158 children 16 years old and below who were considered high risk of having COVID-19 based on family or social contact with someone diagnosed with COVID-19. Of 158 children with possible or suspected COVID-19, clinically, 76 had positive RT-PCR and/or at least one change on $\mathrm{CT}$ that could be compatible with COVID-19 (43/76 [57\%] had positive PCR and positive CT; 7/76 [9\%] had positive RT-PCR but no change on

This comment refers to the article available at https://doi.org/10.1186/ s12916-020-01596-9.

* Correspondence: iansinha@liv.ac.uk

${ }^{1}$ Alder Hey Children's Hospital, Liverpool L12 2AP, UK

${ }^{2}$ Division of Child Health, University of Liverpool, Liverpool, UK
CT; 26/76 [34\%] had changes on CT, but negative RTPCR).

Importantly, the article highlights CT abnormalities which can be found in the majority of children with confirmed COVID-19. In the 43 children who tested positive on RT-PCR and had changes on CT, some radiological trends were observed, and these are similar to changes described in adult chest CT scans. The commonest abnormality was ground-glass opacity, which occurred in 29/43 (67\%), but perhaps more useful is the location of lesions: changes were seen in subpleural regions in $41 / 43(95 \%)$ and in the lower lobes in $28 / 43$ (65\%). The other important finding was that there was a lag in resolution of CT changes as compared with clinical improvement. This is relevant because, at the time the study was conducted, discharge criteria in China included radiological improvement.

So where does this leave the role of CT in children? The answer is that a 'one-size fits all' approach is unlikely to be helpful. For a test to be useful and worthwhile for a patient, at a particular point in time, it should have good diagnostic accuracy, be safe, and have some bearing on clinical decisions.

For children with proven COVID-19 infection but minimal or no respiratory symptoms, we would suggest that chest CT is unlikely to be helpful. In adult COVID-19, which at the time of writing carries a crude in-hospital mortality rate of $30 \%$ in the UK [7], understanding the underlying lung pathology may be useful in determining prognosis or identifying appropriate strategies for respiratory support. In most children with COVID-19, who have self-limiting illness, and mild disease progression, knowing the changes on CT 
is unlikely to guide clinical decisions. For most children with suspected COVID-19, but negative testing, chest CT is also unlikely to be of benefit. If they have mild, selfresolving symptoms, then making the diagnosis is not crucial, and CT (as always) is not a perfect tool for making this diagnosis anyway-as seen in the linked study by $\mathrm{Ma}$ et el., nearly $10 \%$ of children had positive RT-PCR but negative CT [6].

There are particular children in whom CT scanning of the chest may be useful, depending on their clinical status. CT imaging may be useful for children with signs of hyperinflammation. This might reflect an emerging hyperinflammatory phenotype, which bears some resemblance to Kawasaki syndrome that may, at least temporally, be related to the COVID-19 pandemic [8]. In those selected cases, there is an emergent role of CT coronary angiography. CT imaging may be useful in cases of children with suspected ARDS, or hypoxaemia that is disproportionate to the child's dyspnoea. In such cases, CTPA (CT pulmonary angiography) may also be warranted to exclude the possibility of associated pulmonary embolism. In children who are unwell with COVID-19, antiviral medication or targeted immune modulation with anti-interleukin 1 or anti-interleukin 6 therapies may be warranted, preferably within the context of a clinical trial.

When considering the use of diagnostic HRCT scanning for COVID-19 in children, additional factors should be taken into account. Paediatric pulmonary CT scans have been associated with an increased lifetime radiation risk and malignancy [9]. There are logistical difficulties in transporting children to a CT scanner, and the process utilises cleaning and safety equipment that in many settings is in short supply.

\section{Conclusion}

Whilst HRCT changes in children with COVID-19 may appear early in the disease process, we should be circumspect about the routine use of HRCT as a diagnostic modality. There may be a role for this test in some children, but as with all investigations, decisions about when to conduct a CT should be made on a case-by-case basis. One size does not fit all.

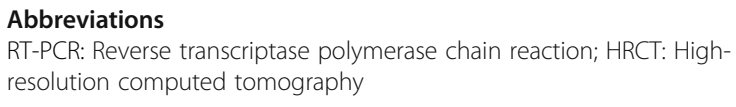

\section{Acknowledgements}

Ms. Rachel Harwood (University of Liverpool) for helping draft the manuscript

\section{Authors' contributions \\ Both authors drafted and agreed the manuscript.}

Funding

No external sources of funding
Availability of data and materials

Not applicable

Ethics approval and consent to participate

Not applicable

Consent for publication

Not applicable

\section{Competing interests}

The authors declare that they have no competing interests.

Received: 20 May 2020 Accepted: 20 May 2020

Published online: 05 June 2020

References

1. Sinha IP, Harwood R, Semple MG, Hawcutt DB, Thursfield R, Narayan O, et al. COVID-19 infection in children. Lancet Respir Med. 2020;8:446-7.

2. Zimmermann P, Curtis N. Coronavirus infections in children including covid19: an overview of the epidemiology, clinical features, diagnosis, treatment and prevention options in children. Pediatr Infect Dis J. 2020;Online First. https://doi.org/10.1097/inf.0000000000002660.

3. Gudbjartsson DF, Helgason A, Jonsson H, Magnusson OT, Melsted P, Norddahl GL, et al. Spread of SARS-CoV-2 in the Icelandic population. N Engl J Med. 2020 https://doi.org/10.1056/NEJMoa2006100.

4. Lee EYP, Ng M-Y, Khong P-L. COVID-19 pneumonia: what has CT taught us? Lancet Infect Dis. 2020. https://doi.org/10.1016/S1473-3099(20)30134-1.

5. Chua F, Armstrong-James D, Desai SR, Barnett J, Kouranos V, Kon OM, et al. The role of $C T$ in case ascertainment and management of COVID-19 pneumonia in the UK: insights from high-incidence regions. Lancet Respir Med. 2020;8:438-40.

6. Ma H, Hu J, Tian J, Zhou X, Li H, Laws MT, et al. A single-center, retrospective study of COVID-19 features in children: a descriptive investigation. BMC Med. 2020;18:123.

7. Docherty AB, Harrison EM, Green CA, Hardwick HE, Pius R, Norman L, et al. Features of 16,749 hospitalised UK patients with COVID-19 using the ISARIC WHO Clinical Characterisation Protocol. preprint. Infect Dis. 2020. https://doi. org/10.1101/2020.04.23.20076042.

8. Riphagen S, Gomez X, Gonzalez-Martinez C, Wilkinson N, Theocharis P. Hyperinflammatory shock in children during COVID-19 pandemic. Lancet. 2020:S0140673620310941.

9. Pearce MS, Salotti JA, Little MP, McHugh K, Lee C, Kim KP, et al. Radiation exposure from $C T$ scans in childhood and subsequent risk of leukaemia and brain tumours: a retrospective cohort study. Lancet. 2012;380:499-505.

\section{Publisher's Note}

Springer Nature remains neutral with regard to jurisdictional claims in published maps and institutional affiliations. 